\section{Die am häufigsten gestellten Fragen zu TarMed}

Kann ich nach Einführung des TarMed als Arzt mit Ausweis "Zweijährige ärztliche Weiterbildung nach KVG" Leistungen mit den Krankenkassen verrechnen? Welcher qualitativen Dignität werde ich zugeordnet?

H. K. in E.

Sie können ausschliesslich Basisgrundleistungen bzw. die mit ihnen abrechenbaren Leistungen (in der Tarifstruktur si bzw. mit pro memoria bezeichnet) verrechnen.

Als prakt. Arzt ohne FMH-Titel bin ich vorwiegend psychiatrisch-psychotherapeutisch in eigener Praxis tätig. Daneben noch, als Überbleibsel meiner früheren hausärztlichen Tätigkeit, in kleinem Umfang als Grundversorger (etwa 5-10\%). Meine Fortbildungspflicht werde ich in Zukunft im psychotherapeutischpsychiatrischen Bereich absolvieren. Bin ich unter TarMed, OHNE zusätzliche Fortbildung als Grundversorger, weiterhin berechtigt, in diesem geringen Umfang Grundversorger-Leistungen zu erbringen?

H. G. in B.
Nein. Jede ärztliche Tätigkeit bedarf für ihre Ausübung einer regelmässigen Fortbildung.

TarMed Pos. 04.0040: Was bedeutet hier "pro Testreihe»?

J. M. in W.

Heisst, dass die erwähnte Abgeltung für die in der Testreihe festgehaltene Zahl von Proben, mit anderen Worten nicht pro einzelne Probe, gilt.

1. Bei kinderkardiologischen Untersuchungen muss naturgemäss häufig eine Pulsoxymetrie durchgeführt werden. Habe diese Position für sich im TarMed nicht gefunden! Bitte um Aufklärung.

2. Wie kann eine Pulsoxymetrie als Einzelleistung bei einer kardiologischen Konsultation verrechnet werden?

P. H. in $B$.

ad 1: Antrag gestellt für Runde 2000.

ad 2: Steht als Antrag für Runde 2000; kann zur Zeit nicht beantwortet werden.

Angegeben wird im TarMed der Ort der Leistungserbringung mit "a1" ambulant, "a2" mehrheitlich stationär und "S" stationär. Einzelne der unter "S" aufgeführten Leistungen werden heute auch ambulant durchgeführt. Heisst die Rubrik "S" nun, dass entsprechende Leistungen nur noch durch ein Spital abgerechnet werden dürfen, auch wenn die Untersuchung ambulant durchgeführt wurde (dies würde ja den bisherigen Spitalleistungskatalog/orangen Tarif

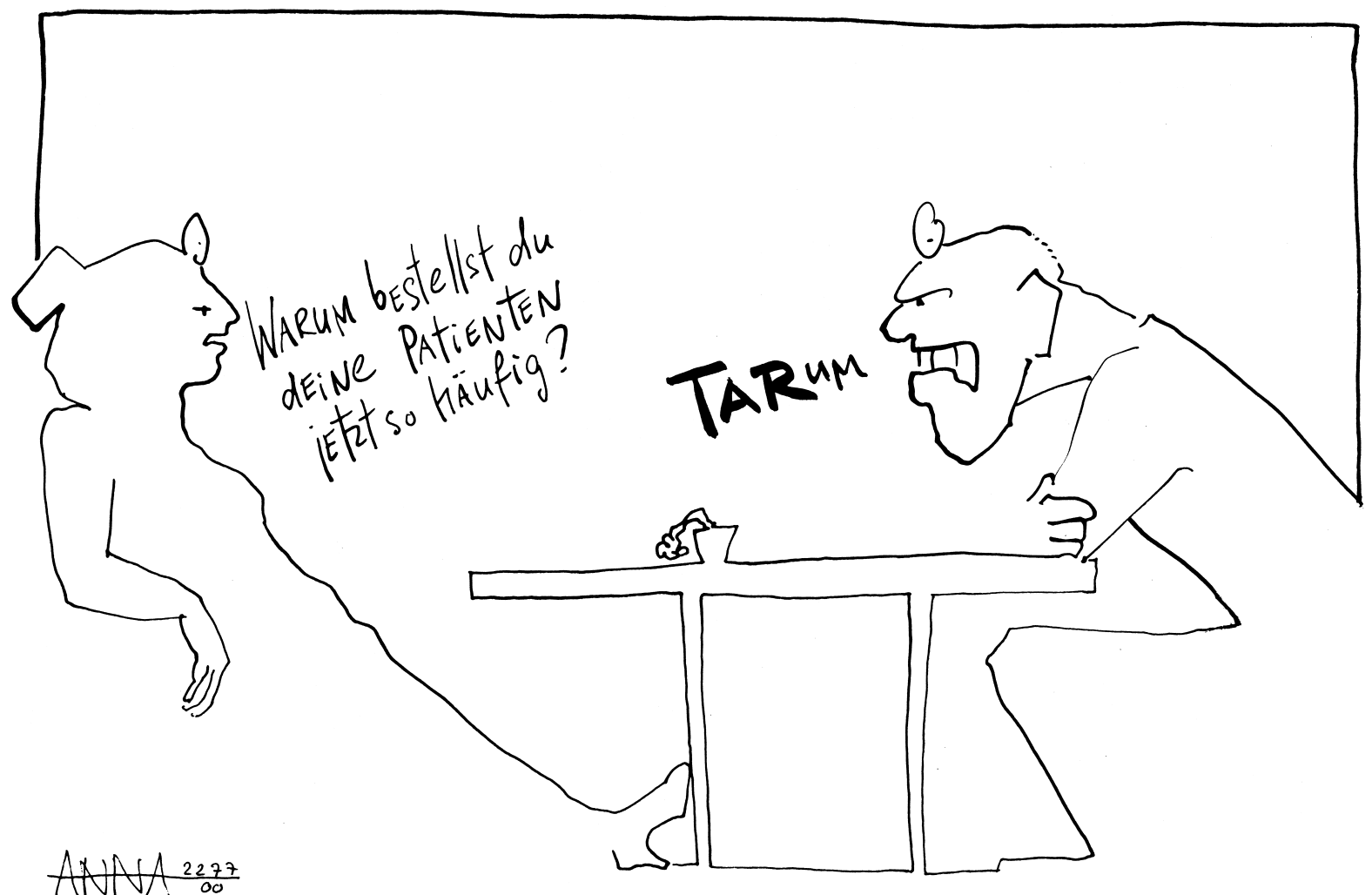


wieder einführen) oder dass die entsprechenden Leistungen ambulant nicht mehr verrechnet werden dürfen (auch nicht mehr ambulant durch Spitäler) sondern immer eine (kurze) Hospitalisation beinhalten?

H. V. in. Z.

S-Leistungen können ambulant verrechnet werden; dies wurde seitens der Versicherer, die eine Verlagerung in den ambulanten Sektor wünschen müssen, so bestätigt. Bitte Leistungen nennen, damit wir Umlagerung in "a2» vornehmen können.

Als Rheumatologe (bisher FMH Physikalische Medizin) habe ich viel in die Physiotherapie investiert. Es ist mir unverständlich, wie die Physiotherapie von der FMH einfach aus dem GRAT rausgeschmissen wurde. Labor und Röntgen sind ja auch keine direkten ärztlichen Leistungen. Genauso wie das Röntgen von der Arztgehilfin ausgeführt wird, machen meine Physiotherapeutinnen gemäss meiner spez. Verordnung die Physiotherapie. Es handelt sich oft um komplexe Fälle, welche schon von den Hausärzten vorbehandelt wurden. Die einzige Alternative wäre die teure Hospitalisation mit stationärer Behandlung. Es scheint sich aufgrund der letzten Informationen herauszukristallisieren, dass die versprochenen Reha-Tarife nur ganz speziellen noch aufwendigeren Fällen in der Reha-Klinik vorbehalten bleiben. Es ist für uns in der Praxis tätigen Rheumatologen so, wie wenn man dem Chirurgen das Skalpell wegnehmen würde. Sollen wir tatsächlich mit dem massiv unterbezahlten Physiotarif abrechnen und uns in den Ruin treiben lassen? Können wir überhaupt mit dem Physiotarif abrechnen, ohne Vertragspartner zu sein? Oder kann man uns dieses Recht ersatzlos streichen? Sollte die FMH nicht wenigstens vertraglich regeln, welche Positionen wir Ärzte in welchen Situationen für komplexe Fälle anwenden dürfen (Pos. 1073)? Oder wird eine Fachgruppe einfach fallengelassen?

A. W. in W.

Physiotherapie war nie im GRAT, konnte also nicht rausgeschmissen werden. Die Leistungen, die durch Arztpraxis verrechenbar sind, werden im Physiotherapietarif gekennzeichnet. Die komplexen Physiotherapieleistungen harren ihrer Tarifierung, Stichwort Tar-Rehab.

Stimmt das Gerücht, dass die FMH weiterhin die Röntgenuntersuchung in der Allgemeinpraxis höher bewerten will (beibehalten der Position für das Einund Ausschalten des Gerätes) als die Untersuchung durch den Spezialisten (FMH Radiologie), auch nach Überarbeitung der Röntgentarife?

R. B. in $B$.

Sie müssen sich nicht auf Gerüchte verlassen; Nachsehen in Version Alpha 2.2 genügt. Zur Abwechslung stimmt dieses Gerücht, natürlich nicht in der von Ihnen formulierten plakativ-provokativen Form: Ausgehend vom Grundsatzentscheid, dass auch in der
Grundversorgerpraxis Nativröntgenleistungen angeboten und entsprechend verrechnet werden sollen, musste eine Sockelleistung für die Grundversorgerpraxis kalkuliert werden, ohne die ein Röntgenbild daselbst nicht mehr nur annähernd kostendeckend angefertigt werden könnte (vgl. Auslastungszahlen).

Es handelt sich hier um einen berufs- und strukturpolitischen Grundsatzentscheid, der tarifarisch umgesetzt werden musste. Für weitere Fusstritte suchen Sie deshalb bitte die richtige Türe auf.

Ich schaue jeden Abend nach der Sprechstunde die Krankengeschichten der Patienten des folgenden Tages durch und bereite mich so gezielt vor. Dies nimmt pro Krankengeschichte zwischen 20 Sekunden und 10 Minuten in Anspruch. Die ganze Arbeit beschäftigt mich jeden Abend 30 bis 40 Minuten und dies 5 Mal pro Woche.

Werde ich nach TarMed für diese Tätigkeit entschädigt?

$$
\text { J. R. in I. }
$$

Diese Tätigkeit ist allenfalls (vorgezogener) Teil der Konsultation, dies aber nur in einem gewissen Ausmass. Arbeit für den Patienten in seiner Abwesenheit kann hier nicht verrechnet werden, solange es um das Studium der eigenen Akten geht.

Als Fachärztin für Allgemeinmedizin und Familienfrau arbeite ich seit vier Jahren teilzeitlich fast ausschliesslich gynäkologisch; den Notfalldienst leiste ich zusammen mit den GrundversorgerInnen, erfülle das Fortbildungsprotokoll der SGAM; meine Fortbildung berücksichtigt allgemeinmedizinische und gynäkologische Themen.

Im TarMed werden die Positionen "gynäkologische Voruntersuchung, gynäkologische Beratung (Pille, Hormone usw.) und Schwangerschaftskontrolle" gemäss qualitativer Dignität den Fachärzten für Gynäkologie und Geburtshilfe zugerechnet.

Meine Fragen:

1. Kann ich weiterhin diese für die Fortführung meiner Praxis existentiellen Positionen abrechnen? (Stichwort Besitzstandswahrung)

2. Welche Instanz entscheidet im Einzelfall über den Punkt "regelmässig erbrachte, bisher unbeanstandete Leistung»?

3. Können Vorsorgeuntersuchungen und Beratungen wirklich nicht mehr vom Grundversorger abgerechnet werden (während zum Beispiel die Positionen Speculum-Untersuchung und Kolposkopie offenbar weiterhin allen offenstehen)?

C. L. in F.

ad 1: Ja.

ad 2: FMH, dann TarMed Nachfolgeorganisation.

ad 3: Kann so ohne Spezifikation nicht beantwortet werden. So sind für gewisse dieser Untersuchungen bundesrechtliche Definitionen und Regelungen vorgegeben, die durch die Vertragsparteien selbstverständlich nicht geändert werden können. 